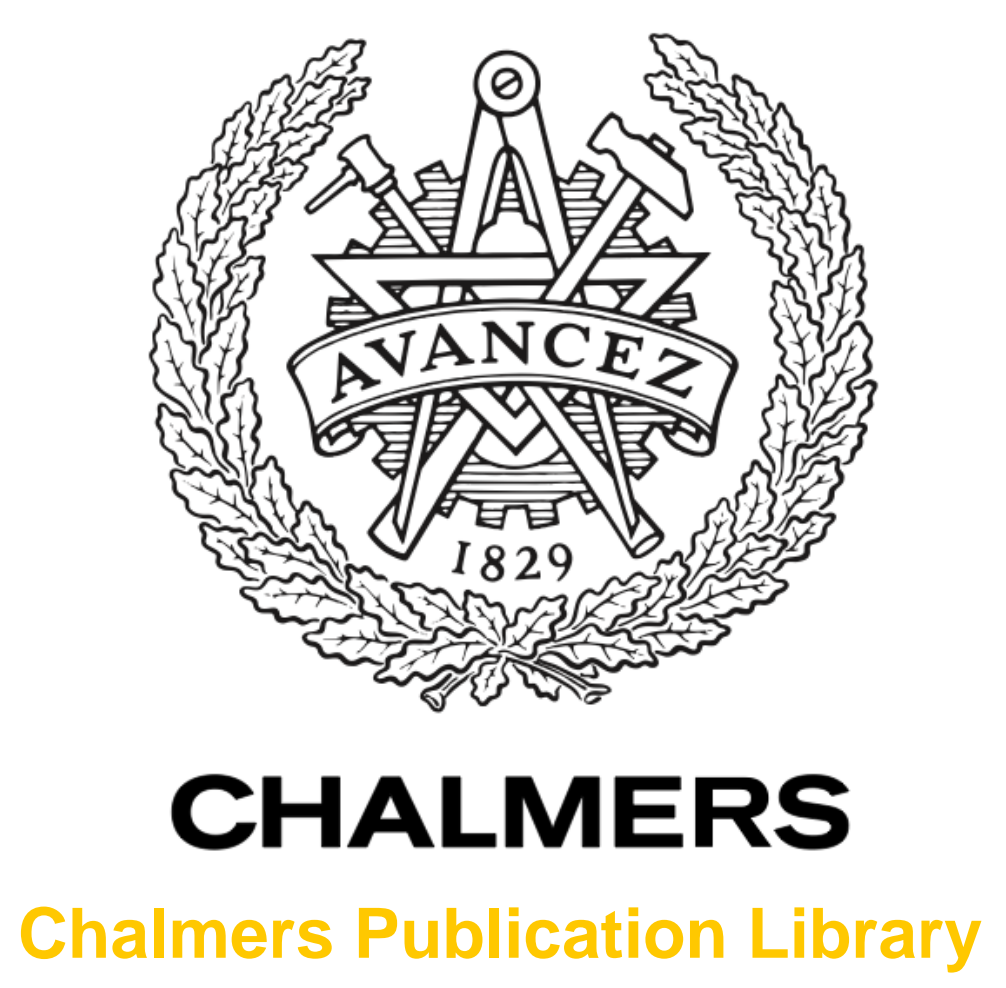

Impact of base station antenna tilt on the performance of network MIMO systems

This document has been downloaded from Chalmers Publication Library $(\mathrm{CPL})$. It is the author's version of a work that was accepted for publication in:

IEEE 75th Vehicular Technology Conference, VTC Spring 2012, Yokohama, 6 May-9 June 2012 (ISSN: 1550-2252)

Citation for the published paper:

Seifi, N. ; Coldrey, M. ; Matthaiou, M. (2012) "Impact of base station antenna tilt on the performance of network MIMO systems". IEEE 75th Vehicular Technology Conference,

VTC Spring 2012, Yokohama, 6 May-9 June 2012

http://dx.doi.org/10.1109/VETECS.2012.6239994

Downloaded from: http://publications.lib.chalmers.se/publication/163664

Notice: Changes introduced as a result of publishing processes such as copy-editing and formatting may not be reflected in this document. For a definitive version of this work, please refer to the published source. Please note that access to the published version might require a subscription.

Chalmers Publication Library (CPL) offers the possibility of retrieving research publications produced at Chalmers University of Technology. It covers all types of publications: articles, dissertations, licentiate theses, masters theses, conference papers, reports etc. Since 2006 it is the official tool for Chalmers official publication statistics. To ensure that Chalmers research results are disseminated as widely as possible, an Open Access Policy has been adopted.

The CPL service is administrated and maintained by Chalmers Library. 


\title{
Impact of Base Station Antenna Tilt on the Performance of Network MIMO Systems
}

\author{
Nima Seifi*, Mikael Coldrey ${ }^{\dagger}$, Michail Matthaiou*, and Mats Viberg* \\ ${ }^{*}$ Department of Signals and Systems, Chalmers University of Technology, Gothenburg, Sweden \\ ${ }^{\dagger}$ Ericsson Research, Ericsson AB, Gothenburg, Sweden \\ Email: \{seifi,michail.matthaiou,viberg\}@chalmers.se, mikael.coldrey@ericsson.com
}

\begin{abstract}
We study the downlink of a multicell MIMO system where clusters of multi-antenna base stations jointly serve multiple single-antenna users, commonly referred to as a network MIMO system. Most of the previous studies on network MIMO have only considered the azimuth pattern of the antenna, while ignoring the elevation pattern. In this paper, we consider both the azimuth and the elevation patterns and investigate the impact of the elevation angle tuning parameter, denoted as the antenna tilt, on the performance of such systems. Using system simulations, it is shown that the promised performance gains of network MIMO systems over conventional non-coordinated systems, crucially depend on the choice of the right tilt setting including the tilt type, i.e., mechanical or electrical, and the tilt angle. In particular, for tilt angles smaller than the optimum, network MIMO with intra-site coordination performs almost as well as the conventional system; while for tilt angles larger than the optimum, the performance of network MIMO with intra-site is similar to that of network MIMO with inter-site coordination.

Index Terms-Antenna tilt, downlink, intercell interference, multiuser MIMO, network MIMO.
\end{abstract}

\section{INTRODUCTION}

Intercell interference is a fundamental performance-limiting factor in the contemporary multicell systems especially for the users at the cell-edge. One of the key strategies to combat this effect is via coordination among multiple base stations, which is currently under investigation in the emerging standards such as LTE-Advanced under the title coordinated multipoint transmission (CoMP) [1]. In one way of realizing CoMP, multiple base stations are inter-connected via backhaul links and process the users data jointly (a.k.a. network MIMO). This results in a significant performance gain compared to the conventional interference-limited systems [2]. Previous studies on network MIMO, however, are only based on 2D antenna models. Those models ignore the elevation pattern and the variable parameter which controls the elevation angle of the antenna, commonly referred to as antenna tilt.

Antenna tilt is one of the most important antenna parameters which affects the intercell interference and the cell coverage [3]. Tilting can be mechanical, electrical, or a combination of the two. Mechanical tilting is achieved via the physical rotation of the antenna and requires site visit. On the other hand, electrical tilting is achieved by changing the phase of the antenna excitation and can be performed remotely by the

This work has been supported in part by VINNOVA within the VINN Excellence Center Chase; and in part by SSF within the Strategic Research Center Charmant. network operator, denoted as remote electrical tilt (RET). RET can adjust the tilt angle at most by $10^{\circ}$ with respect to the normal tilt direction due to the grating lobe effect. This limits the effectiveness of RET if the optimal tilt angle to apply is greater than $10^{\circ}$. In such scenarios, mechanical tilting needs to be combined with RET to realize the optimal angle [3], [4].

Base station antenna tilting has been studied extensively for conventional cellular systems, where cells operate independently and concurrently [3]-[7]. To the best of our knowledge, however, the impact of antenna tilting has not been investigated in the context of network MIMO systems. In this paper, we investigate the effect of antenna tilt on the performance of a network MIMO system in an urban area with a dense deployment of base stations. We consider the long-term average rate (a.k.a. throughput) analysis using three commonly used metrics, namely the cell throughput, the celledge throughput, and the peak throughput. Our simulations results demonstrate that network MIMO achieves the maximum performance gain compared to the conventional system only upon the choice of the right tilt type and the optimum tilt angle. In particular, it is shown that for tilt angles less than the optimum, the performance gain of network MIMO with intra-site coordination over conventional system almost vanishes. For tilt angles larger than the optimum, it is shown that network MIMO with inter-site coordination and intra-site coordination performs very closely.

\section{SySTEM MODEL}

\section{A. Sector Architecture}

In this subsection, we introduce a sector architecture which will serve as a building block for the multicell system considered henceforth. The sector consists of a number of singleantenna users each at a height of $h_{\mathrm{u}}$ above the ground, and a base station equipped with $N_{\mathrm{t}}$ antenna elements at a height of $h_{\text {bs. }}$ An isotropic radiation pattern is assumed for the antenna element at all the users. The radiation pattern for the antenna elements at the base station follows the model proposed by $3 \mathrm{GPP}$ in [8]. The 3GPP antenna pattern is a simple model which has been validated against many commercially available antennas [3]. Based on this model, an antenna coordinate system is defined with Cartesian coordinates $(x, y, z)$ oriented in a way that the $x z$-plane cut of the pattern includes the main lobe peak of the antenna array. The radiation pattern used for 
each antenna element consists of two 1D patterns, namely an azimuth pattern and an elevation pattern. These patterns are formed by a Gaussian shaped main lobe with a side lobe level floor which are defined (in $\mathrm{dBi}$ scale) as ${ }^{1}$

$$
\begin{aligned}
& A_{\mathrm{az}}(\phi)=-\min \left[12\left(\frac{\phi}{\phi_{3 \mathrm{~dB}}}\right)^{2}, S L L_{\mathrm{az}}\right], \\
& A_{\mathrm{el}}(\theta)=-\min \left[12\left(\frac{\theta+\beta_{\mathrm{e}}}{\theta_{3 \mathrm{~dB}}}\right)^{2}, S L L_{\mathrm{el}}\right],
\end{aligned}
$$

where $\beta_{\mathrm{e}}$ is the electrical tilt which is positive when tilting below the $x y$-plane (downtilt) and negative when tilting above it (uptilt), $\phi\left(-180^{\circ} \leq \phi<180^{\circ}\right)$ denotes the azimuth angle relative to the main beam pointing direction and $\theta$ $\left(-90^{\circ} \leq \theta \leq 90^{\circ}\right)$ is the elevation angle relative to the $x y$ plane with $\theta=90^{\circ}$ denoting upwards. Moreover, $\phi_{3 \mathrm{~dB}}=70^{\circ}$ and $\theta_{3 \mathrm{~dB}}=7^{\circ}$ denote the half-power beamwidth (HPBW) in the azimuth and elevation patterns, respectively, while $S L L_{\mathrm{az}}=25 \mathrm{~dB}$ and $S L L_{\mathrm{el}}=20 \mathrm{~dB}$ are the side lobe levels for the respective patterns. The total antenna pattern is determined by the azimuth and elevation patterns according to $[8]$

$$
A(\phi, \theta)=-\min \left[-\left(A_{\mathrm{az}}(\phi)+A_{\mathrm{el}}(\theta)\right), S L L_{0}\right]+A_{0},
$$

where $S L L_{0}=25 \mathrm{~dB}$ is the overall side lobe level and $A_{0}=0$ $\mathrm{dBi}$ denotes the peak antenna gain.

We also define a sector coordinate system with Cartesian coordinates $\left(x^{\prime}, y^{\prime}, z^{\prime}\right)$, which is oriented such that the $x^{\prime} y^{\prime}$ plane is parallel to the ground and the $y^{\prime}$-axis is parallel to the $y$-axis in the antenna coordinate system. Furthermore, all directions in space are defined in terms of the spherical angles $\phi^{\prime}$ and $\theta^{\prime}$ in the sector coordinate system. This is illustrated in Fig. 1(b) for a circular sector with a base station at a point on the perimeter and a sample user with the position vector $\boldsymbol{p}$. Note that $\boldsymbol{p}$ is defined in the sector coordinate system as $\boldsymbol{p}=\left[\begin{array}{lll}p & \phi^{\prime} & \theta^{\prime}\end{array}\right]$ with $p=\|\boldsymbol{p}\|$.

Mechanical tilt is modeled as the rotation of the antenna coordinate system with respect to the sector coordinate system around the $y^{\prime}$-axis. In the case of zero mechanical tilt, the antenna coordinate system coincides with the sector coordinate system (see Fig. 1(b)). Since the antenna pattern is defined in the antenna coordinate system, a transformation is necessary to relate the spherical angles $\left(\phi^{\prime}, \theta^{\prime}\right)$ in the sector coordinate system to the spherical angles $(\phi, \theta)$ in the antenna coordinate system. This transformation is given by [8]

$$
\begin{aligned}
& \theta=\arcsin \left(\cos \phi^{\prime} \cos \theta^{\prime} \sin \beta_{\mathrm{m}}+\sin \theta^{\prime} \cos \beta_{\mathrm{m}}\right), \\
& \phi=\arg \left(\cos \phi^{\prime} \cos \theta^{\prime} \cos \beta_{\mathrm{m}}-\sin \theta^{\prime} \sin \beta_{\mathrm{m}}+j \sin \phi^{\prime} \cos \theta^{\prime}\right),
\end{aligned}
$$

where $\beta_{\mathrm{m}}$ denotes the mechanical tilt angle. It should be noted that mechanical tilting does not change the radiation pattern

\footnotetext{
${ }^{1}$ As for notation, we use lowercase boldface for vectors, uppercase boldface letters for matrices, and calligraphic for sets. $(\cdot)^{\mathrm{H}}, \operatorname{Tr}(\cdot), \mathbb{E}[\cdot],|\mathcal{S}|$, and $\|\boldsymbol{x}\|$ denote Hermitian transpose, trace operator, statistical expectation of a random variable, cardinality of a set $\mathcal{S}$, and the Euclidean norm of $\boldsymbol{x}$, respectively.
}

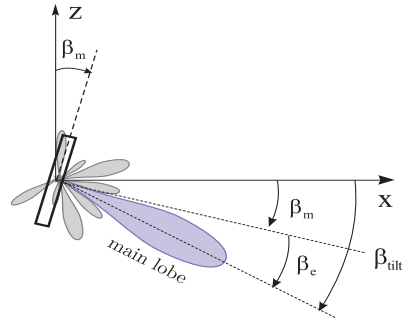

(a)

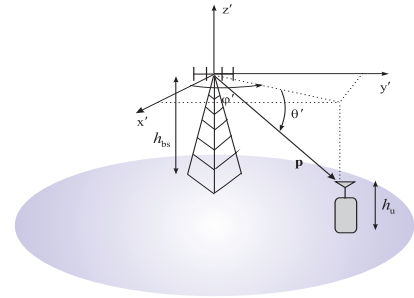

(b)
Fig. 1. Definition of angles and coordinate systems: (a) Illustration of mechanical and electrical tilt; (b) An example of a circular sector architecture.

itself, but rather its projection to the ground. This results in the azimuth pattern and HPBW to depend on the mechanical tilt angle. Electrical tilt, however, creates an elevation steering of the elevation pattern which affects neither the shape of the azimuth pattern nor the azimuth HPBW [3]. Furthermore, with mechanical tilt, the antenna main lobe and back lobe are directed towards opposite directions as the antenna is physically rotated; while in electrical tilt, both main lobe and back lobe move in the same direction, e.g., they are both directed towards the ground when downtilting [7]. Therefore, mechanical and electrical tilting have different impact on the system performance. Finally, the total tilt angle $\beta_{\text {tilt }}$ in the $x z$ plane is given by the sum of electrical and mechanical tilts.

\section{B. Multicell System Architecture}

The clustered cellular network considered here comprises $C$ disjoint clusters, each consisting of $S$ adjacent sectors. To enable coordination, the base stations within the same cluster are connected to a cluster controller via high speed backhaul links, and act as a single distributed multi-antenna transmitter with $S N_{\mathrm{t}}$ antennas. We consider a universal frequency reuse and the downlink transmission over a narrowband frequencyflat fading channel. We focus on the performance of a given cluster, while treating the interference from the other $C-1$ neighboring clusters as noise. The received signal of user $k$ in cluster $c$ is given as

$$
y_{k, c}(t)=\sum_{c^{\prime}=1}^{C} \boldsymbol{h}_{k, c^{\prime}}^{\mathrm{H}}(t) \boldsymbol{x}_{c^{\prime}}(t)+n_{k, c}(t),
$$

where $\boldsymbol{h}_{k, c}(t) \in \mathbb{C}^{S N_{\mathrm{t}} \times 1}$ is the aggregate channel vector between user $k$ and all the base stations in cluster $c, \boldsymbol{x}_{c}(t) \in$ $\mathbb{C}^{S N_{\mathrm{t}} \times 1}$ denotes the aggregate transmit signal vector from all the base stations in cluster $c$, and $n_{k, c}(t) \sim \mathcal{C N}(0,1)$ is the AWGN at user $k$ in cluster $c$. We also assume a sum power constraint of $S$ over all the base stations in the cluster, i.e., $\operatorname{Tr}\left(\mathbb{E}\left[\boldsymbol{x}_{c}(t) \boldsymbol{x}_{c}^{\mathrm{H}}(t)\right]\right) \leq S, \forall c$.

\section{Physical LAyer Signaling AND User SCHEDULING}

To cancel the inter-user interference at each cluster, we focus on linear zero-forcing beamforming (ZFBF) for the 
sake of simplicity. We also assume perfect knowledge of the channel vector of all users in each cluster to all base stations in that cluster at the corresponding cluster controller. Let $K$ denote the total number of users in cluster $c$ with $K \gg S N_{\mathrm{t}}$. Under this assumption, ZFBF cannot serve all the users simultaneously at each time slot. As a result, a scheduler is employed at each cluster controller to select a subset of users to be served at each time slot, referred to as active users. Let $\mathcal{U}_{c}(t)$ denote the set of active users in cluster $c$ at time slot $t$, such that $\left|\mathcal{U}_{c}(t)\right| \leq S N_{\mathrm{t}}$. The transmitted signal $\boldsymbol{x}_{c}(t)$ can then be written as

$$
\boldsymbol{x}_{c}(t)=\sum_{k \in \mathcal{U}_{c}(t)} \boldsymbol{w}_{k, c}(t) d_{k, c}(t),
$$

where $\boldsymbol{w}_{k, c}(t) \in \mathbb{C}^{S N_{\mathrm{t}} \times 1}$ and $d_{k, c}(t)$ denote the beamformer and the data symbol for user $k \in \mathcal{U}_{c}(t)$, respectively. It follows that the transmit covariance matrix at cluster $c$ takes the form

$$
\boldsymbol{Q}_{c}(t)=\mathbb{E}\left[\boldsymbol{x}_{c}(t) \boldsymbol{x}_{c}^{\mathrm{H}}(t)\right]=\sum_{k \in \mathcal{U}_{c}(t)} \boldsymbol{w}_{k, c}(t) \boldsymbol{w}_{k, c}^{\mathrm{H}}(t) p_{k, c}(t),
$$

where $p_{k, c}(t)=\mathbb{E}\left[\left\|d_{k, c}(t)\right\|^{2}\right]$ denotes the allocated power to user $k \in \mathcal{U}_{c}(t)$.

Under ZFBF, the intra-cluster interference is completely removed, i.e., $\boldsymbol{h}_{k, c}^{\mathrm{H}}(t) \boldsymbol{w}_{j, c}(t)=0, \forall j \neq k$. The other cluster interference (OCI) is, however, still present and is treated as noise at each user. The OCI power at user $k$ in cluster $c$ is given by

$$
\chi_{k, c}(t)=\sum_{c^{\prime} \neq c} \boldsymbol{h}_{k, c^{\prime}}^{\mathrm{H}}(t) \boldsymbol{Q}_{c^{\prime}}(t) \boldsymbol{h}_{k, c^{\prime}}(t) .
$$

Now we define the achievable rate $R_{k}\left(\boldsymbol{h}_{k, c}(t), \boldsymbol{Q}_{c}(t), \chi_{k, c}(t)\right)$ of user $k$, expressed in bits/s/Hz, as

$$
\begin{aligned}
R_{k}\left(\boldsymbol{h}_{k, c}(t),\right. & \left.\boldsymbol{Q}_{c}(t), \chi_{k, c}(t)\right) \\
& =\log _{2}\left(1+\frac{\left\|\boldsymbol{h}_{k, c}^{\mathrm{H}}(t) \boldsymbol{w}_{k, c}(t)\right\|^{2} p_{k, c}(t)}{1+\chi_{k, c}(t)}\right) .
\end{aligned}
$$

Using the proportional fairness (PF) algorithm [9], at each time slot $t$ the scheduler at cluster $c$ needs to determine the set of active users $\mathcal{U}_{c}(t)$ and the corresponding transmit covariance matrix $\boldsymbol{Q}_{c}(t)$. The cluster controller then solves the following weighted sum rate maximization problem:

$$
\begin{aligned}
& \max _{\mathcal{U}_{c}(t), \boldsymbol{Q}_{c}(t)} \sum_{k \in \mathcal{U}_{c}} \frac{R_{k}\left(\boldsymbol{h}_{k, c}(t), \boldsymbol{Q}_{c}(t), \chi_{k, c}(t)\right)}{T_{k, c}(t)}, \\
& \text { subject to } \operatorname{Tr}\left(\boldsymbol{Q}_{c}(t)\right) \leq S,
\end{aligned}
$$

where $T_{k, c}(t)$ denotes the windowed long-term average rate of user $k$ at cluster $c$ [9]. The power allocation for a given set $\mathcal{U}_{c}(t)$ is performed using the waterfilling algorithm [10]. For the optimization over $\mathcal{U}_{c}(t)$ we employ the suboptimal, but low-complexity greedy user selection of [11]. We note that $\chi_{k, c}(t)$ in (8) is not known at the scheduling step. To combat this issue, we perform the scheduling based on the average OCI level. We, however, update the long-term average rate using the achievable rate obtained based on the instantaneous ICI power. More details on this approach can be found in [9] and are omitted here for the sake of brevity.

\section{Simulation Setup}

\section{A. Cellular Layout}

A 3-sector hexagonal cell site is considered with the boresight of the array in each sector pointing to the flat side of the sector. We set $N_{\mathrm{t}}=4$ (i.e., 12 antennas per cell site), $h_{\mathrm{bs}}=32$ $\mathrm{m}$, and $h_{\mathrm{u}}=1.5 \mathrm{~m}$. Three different coordination schemes are considered as follows:

- Conventional non-coordinated scheme $(S=1)$ : This corresponds to the case where each cluster consists of only one sector. For multicell simulation, we consider a 19-cell network to account for the two tiers of interfering cells (see Fig. 1.A in [2]).

- Intra-site coordination $(S=3)$ : In this scheme, the three co-located sectors in each cell site form one cluster. The multicell simulation set up is similar to the case of $S=1$.

- Inter-site coordination $(S=9)$ : In this scenario, every 3 adjacent hexagonal cell sites form a cluster. In our multicell simulation, we consider a 21-cell network (see Fig. 1.B in [2]). Note that the network layout for this scenario is different from the other two scenarios as it contains one tier of interfering clusters. Adding more clusters to the layout beyond the first tier, however, has a negligible effect on the statistics of inter-cluster interference, since the interference from those clusters is significantly attenuated due to the increased pathloss [2].

A wraparound technique is employed to avoid any edge effects in the network [9]. In this way, each cluster will observe the same layout irrespective of its position in the network. We define the path gain $\alpha_{k, b, c}$ between user $k$ and base station $b$ in cluster $c$ as

$$
\alpha_{k, b, c}=P_{0}\left(\frac{d_{k, b, c}}{D_{0}}\right)^{-\kappa} 10^{A\left(\phi_{k, b, c}, \theta_{k, b, c}\right) / 10} 10^{\left(v_{k, b, c} / 10\right)},
$$

where $d_{k, b, c}$ denotes the distance between the antenna of user $k$ and the antenna array of base station $b$ in cluster $c$, taking into account the base station and the user heights, $\kappa=3.5$ is the pathloss exponent, while $D_{0}$ is a reference distance. In addition, $P_{0}$ denotes the received power (excluding the antenna gain) at the reference distance $D_{0}, v_{k, b, c}$ is the log-normal shadowing (in $\mathrm{dB}$ ) between user $k$ and base station $b$ in cluster $c$ with a standard deviation of $8 \mathrm{~dB}$, and $A\left(\phi_{k, b, c}, \theta_{k, b, c}\right)$ denotes the base station antenna gain in $\mathrm{dBi}$ at spherical angles $\phi_{k, b, c}$ and $\theta_{k, b, c}$ in the antenna coordinate system. In our simulations, we set the reference distance $D_{0}$ equal to the cell radius (288 $\mathrm{m}$ in this paper), which results in that $P_{0}$ represents the cell-edge SNR, i.e., the SNR achieved at the edge of an isolated cell. Based on the path gain definition, the channel model between user $k$ and base station $b$ in cluster $c$ at time slot $t$ is given by $\sqrt{\alpha_{k, b, c}} \boldsymbol{h}_{k, b, c}(t)$, where $\boldsymbol{h}_{k, b, c}(t) \in \mathbb{C}^{N_{\mathrm{t}} \times 1}$ denotes the small-scale fading channel vector with elements 
$\sim \mathcal{C N}(0,1)$, i.e., isotropic scattering. Note that we assume the distances between the antennas of the same base station to be much smaller than $d_{k, b, c}$. As such, the spherical angles of user $k$ to all the antenna elements in the base station array are almost the same, and all the antennas can be characterized by the same shadowing effects as the shadowing is caused by large scatterers. Finally, the inter-element distance at the base station antenna array is assumed to be sufficiently large so that the effects of spatial correlation can be neglected.

\section{B. Performance Metrics and Simulation Method}

We consider the throughput analysis for the performance evaluation. To this end, three different performance metrics are used, namely the cell throughput, the cell-edge throughput, and the peak throughput defined as the 50-percentile, the 5percentile, and the 95-percentile of the throughput distribution over the cell, respectively. Therefore, we need to obtain the distribution of the throughput over the cell site. We, however, highlight that due to the presence of unknown OCI, multiuser MIMO transmission with PF scheduling, and the non-isotropic statistics of $\boldsymbol{h}_{k, c}(t)$ (and consequently $\left.\boldsymbol{w}_{k, c}(t)\right)^{2}, \forall k, c$, an analytical treatment of the problem under consideration becomes tedious. As a result, we resort to Monte Carlo simulation, and leave the development of an analytical framework to our future work. We use a drop-based simulation, where at each drop 30 users are placed uniformly over the area of each cell site. Each user is then assigned to a cluster based on its maximum path gain to the base station inside that cluster. We assume full intra-site shadowing correlation and an inter-site shadowing correlation of 0.5 .

\section{Numerical Results AND Discussion}

In this section, we compare the performance of the two network MIMO schemes (i.e., intra-site and inter-site coordination) with that of the conventional non-coordinated scheme. Regarding the tilt type, we consider two different tilt types as follows: 1) purely mechanical tilt, and 2) purely electrical tilt. The inter-site distance is $500 \mathrm{~m}$ and the cell-edge SNR is set to $15 \mathrm{~dB}$ to account for an interference-limited system.

In Fig. 2, the cell throughput is plotted versus the tilt angle. By comparing the cell throughput for the two different tilt types in Fig. 2(a), and 2(b), respectively, we observe that for each scheme individually, the maximum cell throughput is achieved by using electrical tilt. It is also observed that the maximum cell throughput gain for network MIMO schemes compared to the conventional scheme is achieved using electrical tilt, and is equal to $\sim 28 \%$ and $\sim 42 \%$ for intra-site and inter-site coordination, respectively.

Figure 3 compares the cell-edge throughput versus the tilt angle. The maximum cell-edge throughput for each scheme individually is achieved via electrical tilt. However, we note that the maximum cell-edge throughput gain for network

\footnotetext{
${ }^{2}$ Since each user $k$ in cluster $c$ experiences different pathloss and shadowing to different base stations in the cluster, the elements of the aggregate channel $\boldsymbol{h}_{k, c}$ have a non-identical distribution.
}

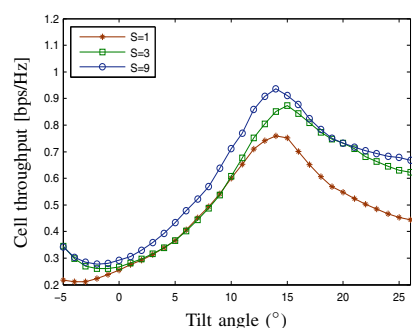

(a) Mechanical tilt

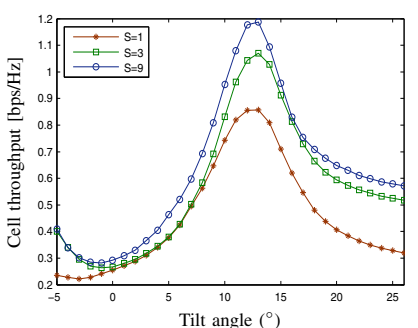

(b) Electrical tilt
Fig. 2. Cell throughput vs. tilt angle for different tilt settings and coordination schemes. $S=1$ denotes the conventional scheme, $S=3$ means intra-site coordination, and $S=9$ is inter-site coordination.

MIMO schemes with respect to the conventional scheme is approximately the same for both mechanical and electrical tilt.

In Fig. 4, the peak throughput versus the tilt angle is plotted. We note that electrical tilt provides negligible peak throughput gain compared to mechanical tilt for the conventional scheme. It is also observed that mechanical tilt provides no peak throughput gain for network MIMO schemes compared to the conventional scheme. Electrical tilt, however, achieves a peak throughput gain of $\sim 20 \%$ for intra-site coordination and $\sim 27 \%$ for inter-site coordination relative to the conventional scheme. It should be noted that the peak throughput is usually achieved by the users close to the base station and to the main beam peak of its antenna array. As a matter of fact, the desired signal of such users is mainly received from this base station.

For the optimum mechanical tilt angle, the desired signal power is the same for both the conventional scheme and the network MIMO schemes, as in both cases the back lobes of the other intra-site base stations are pointing upwards; hence, neither any interference in the conventional scheme nor any desired signal in the network MIMO scheme is received from these back lobes. Furthermore, as the optimum mechanical tilt angle is relatively large, the effect of the signal from the neighboring cells, which is either intercell interference or desired signal in the inter-site coordination case, is negligible for such a user. Therefore, all the schemes have almost the same peak throughput.

For the optimum electrical tilt, however, the back lobes of the other intra-site base stations are also pointing downward. Hence, they act as intra-site interference in the conventional scheme, while contributing to the desired signal in the network MIMO with intra-site coordination, which results in peak throughput increase. Furthermore, as the optimum electrical tilt angle is slightly smaller than the optimum mechanical one, the desired signal component from the adjacent cell in the inter-site coordination case also contributes to the total desired signal resulting in a small improvement compared to the intra-site coordination.

One interesting observation, common in all figures, is that the network MIMO schemes outperform the conventional scheme only over a limited range of tilt angles. Furthermore, the performance of network MIMO schemes is degraded faster 


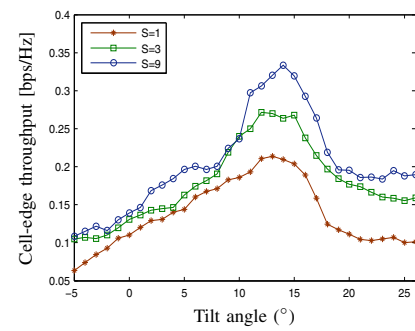

(a) Mechanical tilt

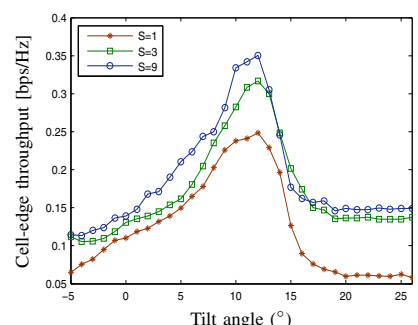

(b) Electrical tilt
Fig. 3. Cell-edge throughput vs. tilt angle for different tilt settings and coordination schemes.

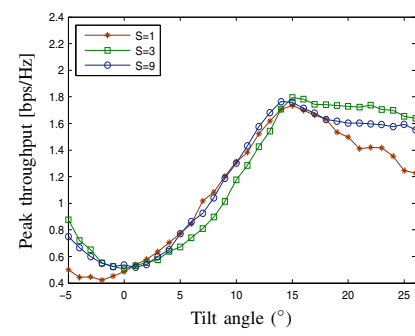

(a) Mechanical tilt

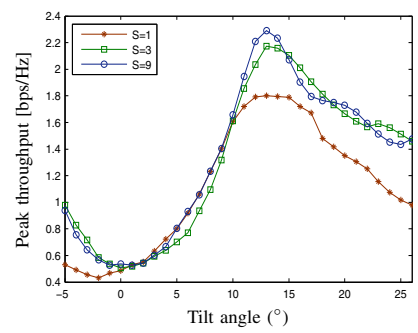

(b) Electrical tilt
Fig. 4. Peak throughput vs. tilt angle for different tilt settings and coordination schemes.

than that of the conventional case when the tilt angle deviates from the optimum, which shows the sensitivity of network MIMO performance with respect to the optimum tilt angle.

Another important point to highlight is that the performance of intra-site coordination gets close to that of the conventional case when the tilt angle is decreased below the optimum, while it gets close to that of inter-site coordination when the tilt angle is increased above the optimum. The reason for the former is that small tilt angles result in severe leakage of interference to the neighboring cells that can easily dominate the intra-site coordination gain. In the latter, however, using large tilt angles isolates the neighboring cells to a large extent such that the effect of the desired signal received from the neighboring cells in inter-site coordination becomes negligible. As a result, most of the gain over conventional system is obtained via the coordination of the co-located base stations inside the cell sites, i.e., intra-site coordination. Furthermore, this coordination gain approaches a constant value for very large tilt angles. In fact, at large tilt angles the cells are highly isolated and the performance is mainly limited by intracell impairments, i.e., noise for the intra-site coordination and intra-cell interference for the conventional system.

Finally, as mentioned before, a commercial RET antenna can typically adjust the tilt angle by at most $10^{\circ}$ with respect to the nominal tilt direction owing to the grating lobe effects [3]. However, the optimal electrical tilt angle in all figures, is greater than $10^{\circ}$ due to the small inter-site distance considered in this paper. Our simulation results have shown that a large fraction of the optimum performance achieved by purely electrical tilting, can also be achieved when the total tilt angle is composed of half electrical and half mechanical tilt angle. Therefore, for scenarios where the optimum tilt angle is larger than the maximum adjustable electrical tilt angle, we can use mechanical tilting to tailor to the optimum with only a marginal loss in performance.

\section{CONCLUSIONS}

We investigated the effect of base station antenna tilt on the performance of a network MIMO system with a dense deployment of base stations. We demonstrated via systemlevel simulations that the throughput gain of network MIMO promised by $2 \mathrm{D}$ models is only achievable upon the selection of the right tilt setting. Purely electrical tilting was shown to offer the best performance for all considered performance metrics and coordination schemes. It was also shown that using a tilt angle smaller than the optimal results in severe leakage of interference to the neighboring cells, thereby degrading the coordination gain. Aggressive tilting, on the other hand, was shown to isolate the cell sites resulting in the intersite coordination gains to diminish. We also observed that the performance of the coordinated schemes degrades faster than that of the conventional scheme, when deviating from the optimal tilt angle. In our future work, we will consider the effect of inter-site distance and sector orientation, e.g., by changing the the bore-sight of the sector array to point to the cell edge.

\section{REFERENCES}

[1] R. Irmer and et al., "Coordinated multipoint: Concepts, performance, and field trial results," IEEE Commun. Mag., vol. 49, no. 2, pp. 102 111, Feb. 2011.

[2] H. Huang and et al., "Increasing downlink cellular throughput with limited network MIMO coordination," IEEE Trans. Wireless Commun., vol. 8, no. 6, pp. 2983-2989, Jun. 2009.

[3] F. Athley and M. N. Johansson, "Impact of electrical and mechanical antenna tilt on LTE downlink system performance," in Proc. of IEEE 71st Veh. Technol. Conf. (VTC), Taipei, Taiwan, May 2010.

[4] O. N. C. Yilmaz, J. Hämäläinen, and S. Hämäläinen, "Self-optimization of remote electrical tilt," in Proc. IEEE Sym. Pers. Indoor Mob. Radio Commun. (PIMRC), Istanbul, Turkey, Sep. 2010.

[5] T. I. Jarno Niemelä and J. Lempiäinen, "Optimum antenna downtilt angles for macrocellular WCDMA network," EURASIP J. Wireless Commun. Netw., vol. 2005, no. 5, p. 12 pages, 2005.

[6] L. Thiele and et al., "Modeling of 3D field patterns of downtilted antennas and their impact on cellular systems," in Proc. ITG Intern. Works. Smart Ant. (WSA), Berlin, Germany, Feb. 2009.

[7] O. Yilmaz, S. Hamalainen, and J. Hamalainen, "Comparison of remote electrical and mechanical antenna downtilt performance for 3GPP LTE," in Proc. IEEE Veh. Technol. Conf. (VTC), Anchorage, Alaska, USA, Sep. 2009.

[8] 3GPP TR 36.814 V9.0, "Further advancements for E-UTRA physical layer aspects," Tech. Rep., Mar. 2010.

[9] S. A. Ramprashad, G. Caire, and H. C. Papadopoulos, "A joint scheduling and cell clustering scheme for MU-MIMO downlink with limited coordination," in Proc. IEEE Int. Conf. Commun. (ICC), Cape Town, South Africa, May 2010.

[10] R. Zhang, "Cooperative multi-cell block diagonalization with per-basestation power constraints," IEEE J. Sel. Areas Commun., vol. 28, no. 9 , pp. 1435-1445, Dec. 2010.

[11] G. Dimic and N. D. Sidiropoulos, "On downlink beamforming with greedy user selection: performance analysis and a simple new algorithm," IEEE Trans. Signal Process., vol. 53, no. 10, pp. 3857-3868, Oct. 2005. 